\title{
Some Curvature Properties on a Special Paracontact Kenmotsu Manifold with Respect to Semi-Symmetric Connection
}

\author{
K. L. Sai Prasad ${ }^{1, *}$, T. Satyanarayana ${ }^{2}$ \\ ${ }^{1}$ Department of Mathematics, Gayatri Vidya Parishad College of Engineering for Women, Visakhapatnam, Andhra Pradesh, India \\ ${ }^{2}$ Department of Mathematics, Pragathi Engineering College, Surampalem, Near Peddapuram, Andhra Pradesh, India \\ *Corresponding author: klsprasad@yahoo.com
}

Received February 11, 2015; Revised August 23, 2015; Accepted September 17, 2015

\begin{abstract}
The object of the present paper is to study some properties of curvature tensor $\tilde{R}$ of a semi-symmetric non-metric connection $\tilde{\nabla}$ in a type of special paracontact Kenmotsu (briefly SP-Kenmotsu) manifold. We have deduced the expressions for curvature tensor $\tilde{R}$ and the Ricci tensor $\tilde{S}$ of $\mathrm{M}_{\mathrm{n}}$ with respect to semi-symmetric nonmetric connection $\tilde{\nabla}$. It is proved that in an SP-Kenmotsu manifold if the curvature tensor of the semi-symmetric non-metric connection vanishes then the manifold is projectively flat.
\end{abstract}

Keywords: curvature tensor, ricci tensor, projective curvature tensor, non-metric connection, sp-kenmotsu manifold

Cite This Article: K. L. Sai Prasad, and T. Satyanarayana, "Some Curvature Properties on a Special Paracontact Kenmotsu Manifold with Respect to Semi-Symmetric Connection.” Turkish Journal of Analysis and Number Theory, vol. 3, no. 3 (2015): 94-96. doi: 10.12691/tjant-3-4-1.

\section{Introduction}

Friedmann and Schouten [1,2] introduced the idea of semi-symmetric linear connection on a differentiable manifold. Hayden [3] introduced semi-symmetric metric connection on a Riemannian manifold and it was further developed by Yano [4]. Semi-symmetric connections play an important role in the study of Riemannian manifolds. There are various physical problems involving the semisymmetric metric connection. For example, if a man is moving on the surface of the earth always facing one definite point, say Jaruselam or Mekka or the North pole, then this displacement is semi-symmetric and metric [1]. In 1975, Prvanovi'c [5] introduced the concept of semisymmetric non-metric connection with the name pseudometric, which was further studied by Andonie [6,7]. The study of semi-symmetric non-metric connection is much older than the nomenclature 'non-metric' was introduced. In 1992, Agashe and Chafle [8] introduced a semisymmetric connection $\tilde{\nabla}$ satisfying $\tilde{\nabla}_{X} g \neq 0$ on a Riemannian manifold, and called such a connection as semi-symmetric non-metric connection. Later, the curvature properties of the connection in an SP-Sasakian manifold were studied by Bhagwat Prasad [9], and many others.

On the other hand, in 1976, Sato [10] defined the notions of an almost paracontact Riemannian manifold. After that, T. Adati and K. Matsumoto [11] defined and studied para-Sasakian and SP-Sasakian manifolds which are regarded as a special kind of an almost contact Riemannian manifolds. Before Sato, in 1972, Kenmotsu
[12] defined a class of almost contact Riemannian manifolds satisfying some special conditions. In 1995, Sinha and Sai Prasad [13] have defined a class of almost paracontact metric manifolds namely para Kenmotsu (briefly P-Kenmotsu) and special para Kenmotsu (briefly SP-Kenmotsu) manifolds.

In 1970, Pokhariyal and Mishra [14] have introduced new tensor fields, called $\mathrm{W}$ and E-tensor fields in a Riemannian manifold and studied their properties. In the present paper, we consider the W-curvature tensor of a semi-symmetric non-metric connection and obtained a relation connecting the curvature tensors of $\mathrm{Mn}$ with respect to semi-symmetric non-metric connection and the Riemannian connection. It is proved that in an SPKenmotsu manifold if the curvature tensor of the semisymmetric non-metric connection vanishes then the manifold is protectively flat.

Let $\mathrm{M}_{\mathrm{n}}$ be an $\mathrm{n}$-dimensional differentiable manifold equipped with structure tensors ( $\Phi, \xi, \eta$ ) where $\Phi$ is a tensor of type $(1,1), \xi$ is a vector field, $\eta$ is a 1 -form such that

$$
\begin{gathered}
\eta(\xi)=1 \\
\Phi^{2}(X)=X-\eta(X) \xi ; \bar{X}=\Phi X
\end{gathered}
$$

Then $\mathrm{M}_{\mathrm{n}}$ is called an almost paracontact manifold.

Let $\mathrm{g}$ be the Riemannian metric in an n-dimensional almost paracontact manifold $\mathrm{M}_{\mathrm{n}}$ such that

$$
\begin{gathered}
g(X, \xi)=\eta(X) \\
\Phi \xi=0, \eta(\Phi X)=0, \operatorname{rank} \Phi=n-1 \\
g(\Phi X, \Phi Y)=g(X, Y)-\eta(X) \eta(Y)
\end{gathered}
$$


for all vector fields $X$ and $Y$ on $M_{n}$. Then the manifold $M_{n}$ [10] is said to admit an almost paracontact Riemannian structure ( $\Phi, \xi, \eta, g$ ) and the manifold is called an almost paracontact Riemannian manifold.

A manifold $\mathrm{M}_{\mathrm{n}}$ with Riemannian metric ' $\mathrm{g}$ ' admitting a tensor field $\Phi$ of type $(1,1)$, a vector field $\xi$ and 1 -form $\eta$ satisfying equations (1.1), (1.3) along with

$$
\begin{gathered}
\left(\nabla_{X} \eta\right) Y-\left(\nabla_{Y} \eta\right) X=0 \\
\left(\nabla_{X} \nabla_{Y} \eta\right) Z=[-g(X, Z)+\eta(X) \eta(Z)] \eta(Y) \\
+[-g(X, Y)+\eta(X) \eta(Y)] \eta(Z) \\
\nabla_{X} \xi=\Phi^{2} X=X-\eta(X) \xi
\end{gathered}
$$

is called a para Kenmotsu manifold or briefly P-Kenmotsu manifold [13], where $\nabla$ is the covariant differentiation with respect to g.

It is known that [13] on a P-Kenmotsu manifold the following relations hold:

$$
\begin{gathered}
\operatorname{Ric}(X, \xi)=-(n-1) \eta(X) \\
g[R(X, Y) Z, \xi]=\eta[R(X, Y, Z)] \\
=g(X, Z) \eta(Y)-g(Y, Z) \eta(X)
\end{gathered}
$$

where $\mathrm{R}$ is the Riemannian curvature.

Let $\left(M_{n}, g\right)$ be an n-dimensional Riemannian manifold admitting a tensor field $\Phi$ of type $(1,1)$, a vector field $\xi$ and 1-form $\eta$ satisfying

$$
\begin{aligned}
& \qquad\left(\nabla_{X} \eta\right) Y=g(X, Y)-\eta(X) \eta(Y) \\
& g(X, \xi)=\eta(X) \text { and }\left(\nabla_{X} \eta\right) Y=\phi(\bar{X}, Y), \\
& \text { where } \phi \text { is an associate of } \Phi
\end{aligned}
$$

is called a special para Kenmotsu manifold or briefly SPKenmotsu manifold [13].

A linear connection $\tilde{\nabla}$ in a Riemannian manifold $\mathrm{M}_{\mathrm{n}}$ is said to be semi-symmetric connection if its torsion tensor $\mathrm{T}$ satisfies

$$
T(X, Y)=\eta(Y) X-\eta(X) Y
$$

A semi-symmetric non-metric connection $\tilde{\nabla}$ in an almost paracontact metric manifold with torsion tensor (1.13) is given by

$$
\tilde{\nabla}_{X} Y=\nabla_{X} Y+\eta(Y) X
$$

where $\nabla$ is a Riemannian connection with respect to metric g [8].

Apart from conformal curvature tensor, the projective curvature tensor is an other important tensor from the differential geometric point of view. The Weyl-projective curvature tensor $\mathrm{W}$ of type $(1,3)$ of a Riemannian manifold $M_{n}$ with respect to the Riemannian connection is defined by [14]

$$
W(X, Y) Z=R(X, Y) Z-\frac{1}{n-1}\left[\begin{array}{l}
\operatorname{Ric}(Y, Z) X \\
-\operatorname{Ric}(X, Z) Y
\end{array}\right]
$$

for $\mathrm{X}, \mathrm{Y}, \mathrm{Z} \in T(M)$, where $\mathrm{R}$ is the curvature tensor and Ric is the Ricci tensor. If there exists a one-to-one correspondence between each coordinate neighbourhood of a Riemannian manifold $\mathrm{M}_{\mathrm{n}}$ and a domain in Eucledian space such that any geodesic of the Riemannian manifold corresponds to a straight line in the Eucledian space, then $M_{n}$ is said to be locally projectively flat. For $n \geq 3, M_{n}$ is locally projectively flat if and only if the projective curvature tensor $\mathrm{W}$ vanishes. For $\mathrm{n}=2$, the projective curvature tensor identically vanishes.

\section{Curvature Tensor}

The manifold $M_{n}$ is considered to be an SP-Kenmotsu manifold. Let us denote the curvature tensor of the semisymmetric non-metric connection $\tilde{\nabla}$ by $\tilde{R}$ and the curvatre tensor of $\nabla$ by R. By straight forward calculation, we get

$$
\begin{aligned}
& \tilde{R}(X, Y, Z) \\
& =R(X, Y, Z)+\left(\tilde{\nabla}_{X} \eta\right)(Z) Y-\left(\tilde{\nabla}_{Y} \eta\right)(Z) X .
\end{aligned}
$$

As a consequence of equations (1.11) and (1.14), equation (2.1) reduces to

$$
\tilde{R}(X, Y, Z)=R(X, Y, Z)+g(X, Z) Y-g(Y, Z) X
$$

which is the relation between the curvature tensors of $M_{n}$ with respect to the semi-symmetric non-metric connection $\tilde{\nabla}$ and the Riemannian connection $\nabla$.

It is well known that a Riemannian manifold is of constant curvature if and only if it is projectively flat or conformally flat [15] and in general, the necessary and sufficient condition for a manifold with a symmetric linear connection to be projectively flat is that the projective curvature tensor with respect to it vanishes identically on a manifold [16].

As an example, if $M_{n}$ is a Riemannian manifold with vanishing curvature tensor with respect to semi-symmetric non-metric connection, then $M_{n}$ is projectively flat [8]. Analogus to this, we prove the following for an SP-Kenmotsu manifold which is Riemannian.

Theorem 2.1: If in an SP-Kenmotsu manifold $M_{n}$ the curvature tensor of a semi-symmetric non-metric connection $\tilde{\nabla}$ vanishes, then the manifold is projectively flat.

Proof: Since $\tilde{R}=0$, then equation (2.2) gives

$$
R(X, Y, Z)=g(Y, Z) X-g(X, Z) Y \text {. }
$$

On contracting the above equation, we get

$$
\operatorname{Ric}(Y, Z)=(n-1) g(Y, Z) \text {. }
$$

Then, by (2.3) and (2.4), we get

$$
R(X, Y, Z)-\frac{1}{n-1}[\operatorname{Ric}(Y, Z) X-\operatorname{Ric}(X, Z) Y]=0
$$

or $\mathrm{W}=0$ from (1.15), proves that the manifold is projectively flat.

Theorem 2.2: If in an SP-Kenmotsu manifold the Ric tensor of a semi-symmetric non-metric connection $\tilde{\nabla}$ vanishes, then the curvature tensor of $\tilde{\nabla}$ is equal to the projective curvature tensor of the manifold $M_{n}$.

Proof: From equation (2.2), we have

$$
\begin{aligned}
& \tilde{R}(X, Y, Z, U) \\
= & R(X, Y, Z, U)+g(X, Z) g(Y, U)-g(Y, Z) g(X, U) .
\end{aligned}
$$


On contracting the above equation, we get

$$
\text { 'Ric }(Y, Z)=\operatorname{Ric}(Y, Z)-(n-1) g(Y, Z) \text {. }
$$

Since 'Ric $=0$, we have

$$
g(Y, Z)=\frac{1}{n-1}[\operatorname{Ric}(Y, Z)] .
$$

From equations (2.2) and (2.8), we have $\tilde{R}=\mathrm{W}$.

Theorem 2.3: In an SP-Kenmotsu manifold the projective curvature tensor of a semi-symmetric non-metric connection $\tilde{\nabla}$ is equal to the projective curvature tensor of the manifold.

Proof: From equations (2.2) and (2.7), we get

$$
\begin{gathered}
\tilde{R}(X, Y, Z)=R(X, Y, Z)+\frac{1}{n-1}\left[\begin{array}{c}
' \operatorname{Ric}(Y, Z) \\
-\operatorname{Ric}(Y, Z)
\end{array}\right] X \\
-\frac{1}{n-1}[\text { Ric }(X, Z)-\operatorname{Ric}(X, Z)] Y .
\end{gathered}
$$

The terms of the equation (2.9) can be rearranged as

$$
\begin{aligned}
& \tilde{R}(X, Y, Z)-\frac{1}{n-1}[' \operatorname{Ric}(Y, Z) X-' \operatorname{Ric}(X, Z) Y] \\
& =R(X, Y, Z)-\frac{1}{n-1}[\operatorname{Ric}(Y, Z) X-\operatorname{Ric}(X, Z) Y]
\end{aligned}
$$

which is ' $\mathrm{W}=\mathrm{W}$, where ' $\mathrm{W}$ is the Weyl projective curvature tensor with respect to the semi-symmetric nonmetric connection.

Theorem 2.4: In an SP-Kenmotsu manifold with semisymmetric non-metric connection $\tilde{\nabla}$ we have
a) $\tilde{R}(X, Y, Z)+\tilde{R}(Y, Z, X)+\tilde{R}(Z, X, Y)=0$
b) ' $\tilde{R}(X, Y, Z, U)+$ ' $\tilde{R}(X, Y, U, Z)=0$

Proof: Using the Bianchi's first identity with respect to the Riemannian connection equation (2.2) gives (a). From equation (2.6) we get (b).

\section{Acknowledgement}

The authors acknowledge Prof. Kalpana, Banaras Hindu University, Dr. B. Satyanarayana of Nagarjuna University and Dr. A. Kameswara Rao, G.V.P. College of Engineering for Women for their valuable suggestions in preparation of the manuscript. They are also thankful to the referee for his valuable comments in the improvement of this paper.

\section{Competing Interest}

The authors declare that there is no conflict of interests regarding the publication of this paper.

\section{References}

[1] Friedmann and Schouten, J. A., Uber die Geometrie der halbsymmetrischen Ubertragungen, Math Zeitschrift, 21, 211-223, 1924.

[2] Schouten, J. A., Ricci-calculus, Springer-Verlag. Berlin, 1954.

[3] Hayden, H. A., Subspaces of a space with torsion, Proc. London Math. Soc., 34, 27-50, 1932.

[4] Yano, K., On semi-symmetric metric connection, Revue Roumanine de Mathematiques Pures et Appliques, 15, 1579-1581, 1970.

[5] Prvanovi'c, M., On pseudo metric semi-symmetric connections, Pub. De L'Institut Math., N.S., 18(32), 157-164, 1975.

[6] Andonie, P.O.C., On semi-symmetric non-metric connection on a Riemannian manifold. Ann. Fac. Sci. De Kinshasa, Zaire sect. Math. Phys., 2, 1976.

[7] Andonie, P.O.C., Smaranda, D., Certains connections semisymmetriques, Tensor (N.S.), 31, 8-12, 1977.

[8] Agashe, N. S. and Chafle, M. R., A semi-symmetric non-metric connection on a Riemannian manifold, Ind. J. of Pure and Appl. Math., 23(6), 399-409, 1992.

[9] Prasad, On a semi-symmetric non-metric connection in an spSasakian manifold, Istambul Univ.Fen Fak. Mat. Der., 53, 77-80, 1994.

[10] Sato, I., On a structure similar to the almost contact structure, Tensor (N.S.), 30, 219-224, 1976.

[11] Adati, T. and Matsumoto, K., On conformally recurrent and conformally symmetric P-Sasakian manifolds, TRU Math., 13, 2532, 1977.

[12] Kenmotsu, K., A class of almost contact Riemannian manifolds, Tohoku Math. Journal, 24, 93-103, 1972.

[13] Sinha, B. B. and Sai Prasad, K. L., A class of almost para contact metric Manifold, Bulletin of the Calcutta Mathematical Society, 87, 307-312, 1995.

[14] Pokhariyal, G.P., Mishra, R.S., The curvature tensors and their relativistic significance, Yokohoma Math.J., 18, 105-108, 1970.

[15] Yano, K. and Bochner, S., Curvature and Betti numbers, Annals of Math Studies 32, Princeton University Press, 1953.

[16] Sinha, B. B., An introduction to modern Differential Geometry, Kalyani Publishers, New Delhi, 1982. 